\title{
Fourier Transform Scanning Tunneling Spectroscopy: A New Window on the Electronic Structure of $\mathrm{Bi}_{2} \mathrm{Sr}_{2} \mathrm{CaCu}_{2} \mathrm{O}_{8+\delta}$
}

\author{
J.C. DAVIS \\ Department of Physics, LASSP, Cornell University \\ Ithaca, NY 14850, USA
}

\begin{abstract}
Scanning tunneling spectroscopy is used to study the dispersive wave vectors of the local-density-of-states modulations in near-optimal $\mathrm{Bi}_{2} \mathrm{Sr}_{2} \mathrm{CaCu}_{2} \mathrm{O}_{8+\delta}$. Atomic-resolution energy-resolved spectroscopic images are acquired in a $650 \AA$ field of view on the $\mathrm{BiO}$ cleave surface of these crystals at $4.2 \mathrm{~K}$. Fourier transforms are used to measure the wave vectors of spatial modulations in the local density of states. At sub-gap energies, up to 16 inequivalent sets of dispersive wave vectors are observed. When analyzed within a model of quasiparticle scattering-induced interference between a characteristic "octet" of states in momentum-space, they yield an estimate of the Fermi-surface location and the energy gap $|\Delta(k)|$ in agreement with angle-resolved photoemission spectroscopy. At energies approaching the gap-maximum, the local-density-of-states modulations become intense, commensurate with the crystal, and localized by the apparent nanoscale domains. This may indicate that the lifetimes of the $k=\left(\pi / a_{0}, 0\right)$ quasiparticles are determined by nanoscale disorder scattering.
\end{abstract}

PACS numbers: 74.72.-h, 68.37.Ef

\section{High- $T_{\mathrm{c}}$ superconductivity}

The parent compounds of the cuprate high temperature superconductors are antiferromagnetic Mott insulators. These crystals contain 2-dimensional planes consisting of square $\mathrm{CuO}_{2}$ unit cells with dimension $a_{0} \approx 3.8 \AA$. Standard band theory would predict a metallic half-filled electronic band. However, these materials are antiferromagnetic insulators with $\mathrm{a} \approx 3 \mathrm{eV}$ energy gap due to strong correlations. New electronic states are created in this gap when the materials are doped using different valence atoms. A schematic phase diagram of the cuprates is shown in Fig. 1. The maximum critical temperature $T_{\mathrm{C}}$ of any cuprate superconductor 


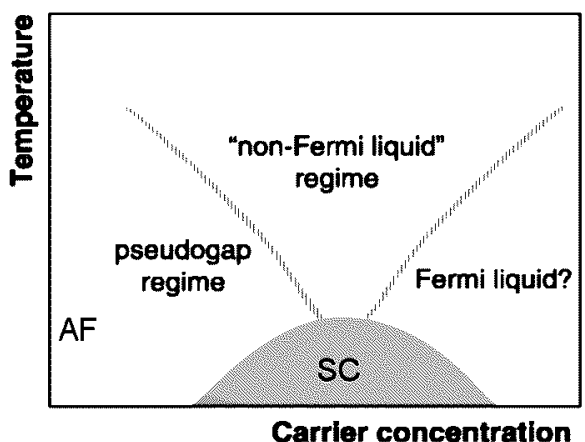

Fig. 1. A schematic representation of the hole-doped copper-oxide phase diagram adapted from Ref. [1].

is $\approx 150 \mathrm{~K}$ and this $T_{\mathrm{c}}$-maximum occurs at a hole-doped carrier concentration of $0.16 \pm 1$ holes per $\mathrm{CuO}_{2}$ plaquette. Among the key challenges to understanding the cuprates are the identification of the electronic ground states and associated excited states, for each part of this phase diagram.

\section{2. $\mathrm{Bi}_{2} \mathrm{Sr}_{2} \mathrm{CaCu}_{2} \mathrm{O}_{8+\delta}$}

A widely studied cuprate is $\mathrm{Bi}_{2} \mathrm{Sr}_{2} \mathrm{CaCu}_{2} \mathrm{O}_{8+\delta}$ (Bi-2212). Its maximum $T_{c}$ is near $95 \mathrm{~K}$. The unit cell structure contains two $\mathrm{Bi}-\mathrm{O}$ planes which are charge neutral and weakly mechanically coupled. This means that $\mathrm{Bi}_{2} \mathrm{Sr}_{2} \mathrm{CaCu}_{2} \mathrm{O}_{8+\delta}$ can be easily cleaved between these two planes to reveal a $\mathrm{BiO}$ surface. The quality and reliability of this cleaved $\mathrm{BiO}$ surface has resulted in its wide use for angle-resolved photoemission spectroscopy (ARPES) studies [2-4]. For the same reasons this system has also been used for STM studies. Figure $2 \mathrm{~B}$ and $\mathrm{C}$ show typical topographic images of this $\mathrm{BiO}$ surface taken from our studies.

On these surfaces the $\mathrm{Bi}$ atoms are clearly seen. In addition, there is a corrugation of $\approx 26 \AA$ period occurring at $45^{\circ}$ to the $\mathrm{Cu}-\mathrm{O}$ bonds. This is the manifestation at the $\mathrm{BiO}$ surface of the three-dimensional crystal supermodulation. Nanoscale electronic disorder [5, 6] is also manifested as the few nm length scale light and dark patches where the tip moves forward and backward apparently to compensate for variations in integrated density of states.

\section{Bi-2212 momentum-space electronic structure and ARPES}

Angle-resolved photoemission has been used with great success to study the cuprate electronic structure [2-4] especially in Bi-2212. In this technique, the electronic spectral function $A(k, \omega)$ is determined from the intensity of photoemission at momentum transfer $p=\hbar k$ and energy transfer $\omega$. Using ARPES, the location of the Fermi surface $[7,8]$, and the magnitude of the energy gap in $k$-space $|\Delta(\boldsymbol{k})|[9-12]$ of $\mathrm{Bi}-2212$ have been determined. 

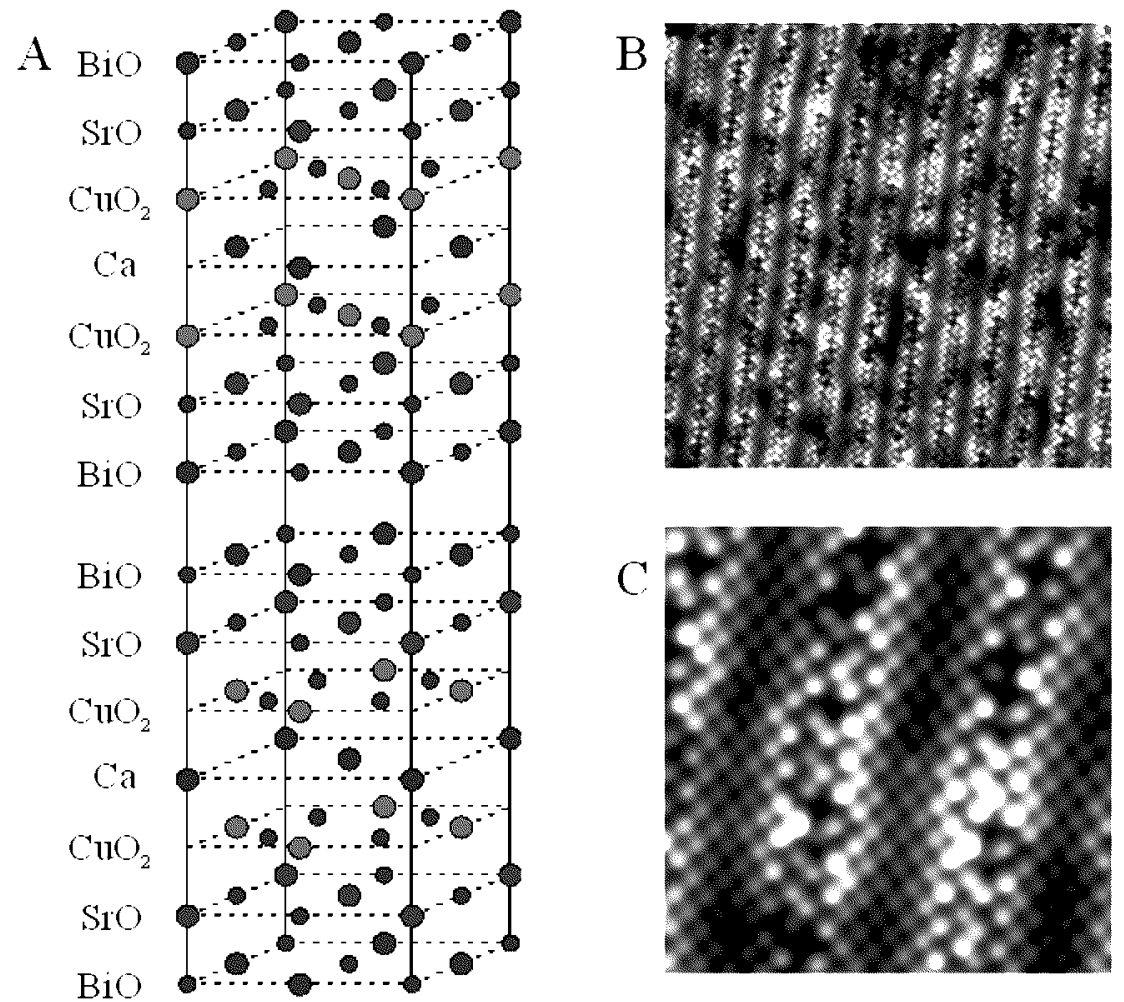

Fig. 2. (A) A schematic representation of the Bi-2212 unit cell. (B) A $250 \AA$ square topographic image of $\mathrm{BiO}$ layer. (C) A $65 \AA$ square topographic image of $\mathrm{BiO}$ layer.
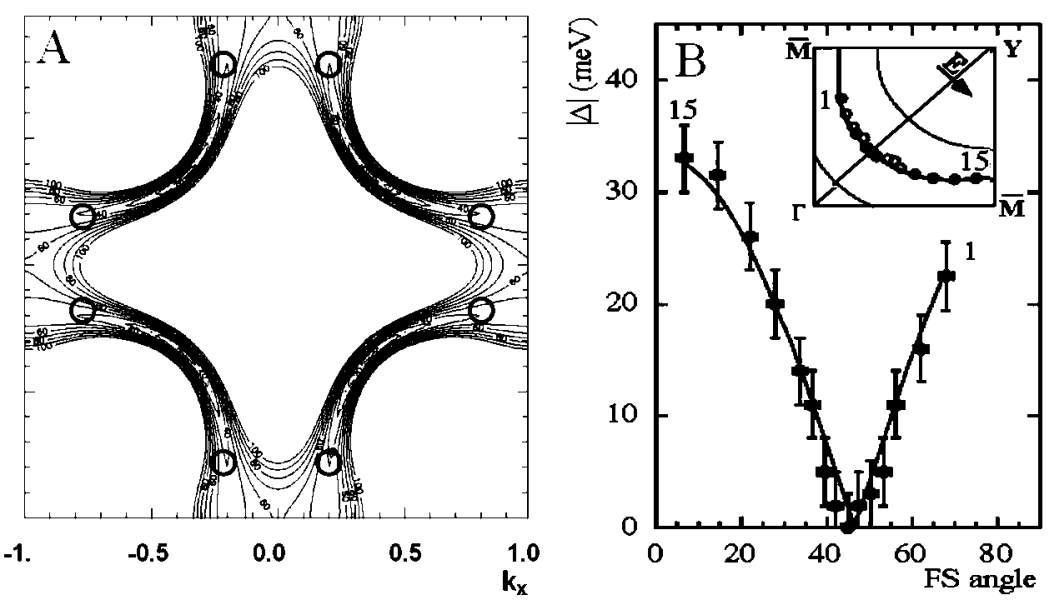

Fig. 3. (A) A parameterization of the superconducting electronic band-structure as deduced from ARPES. (B) Measured structure of the anisotropic energy gap along the Fermi surface. 
In Fig. 3A we show as solid lines the locus in momentum space of quasiparticle states with different energies ranging from $E=-100 \mathrm{meV}$ (below the Fermi level) to $E=+100 \mathrm{meV}$. For energies less than the gap maximum $\Delta_{0}$, these "contours of constant energy" are banana shaped. This parameterization of the electronic structure in momentum-space has been deduced $[2,3]$ from ARPES experiments on the filled states of Bi-2212.

In Fig. 3B we show the magnitude of the superconducting energy as a function of momentum-space location along the trajectory of the normal state Fermi surface (see Ref. [3] for details). From it we see that there are four nodes in the gap at the intersections of the 1 st Brillouin zone diagonals and the normal-state Fermi surface.

\section{Scanning tunneling spectroscopy techniques}

In STM-based tunneling spectroscopy, the tip-sample differential tunneling conductance $g=\mathrm{d} I / \mathrm{d} V$ is measured over a range of sample bias voltages $V$. The result, $g(V)$, is proportional to the local density of states, $\operatorname{LDOS}(E)$ at energy $E=e V$. For a system with perfect $d$-wave BCS superconductivity with momentum dependence of energy gap as shown in Fig. 4A, the expected tunneling spectrum at a single point on the surface is shown in Fig. 4B. Figure 4C is the typical spectrum observed on optimally doped $\mathrm{Bi}-2212$ samples and is in reasonable agreement with expectations.
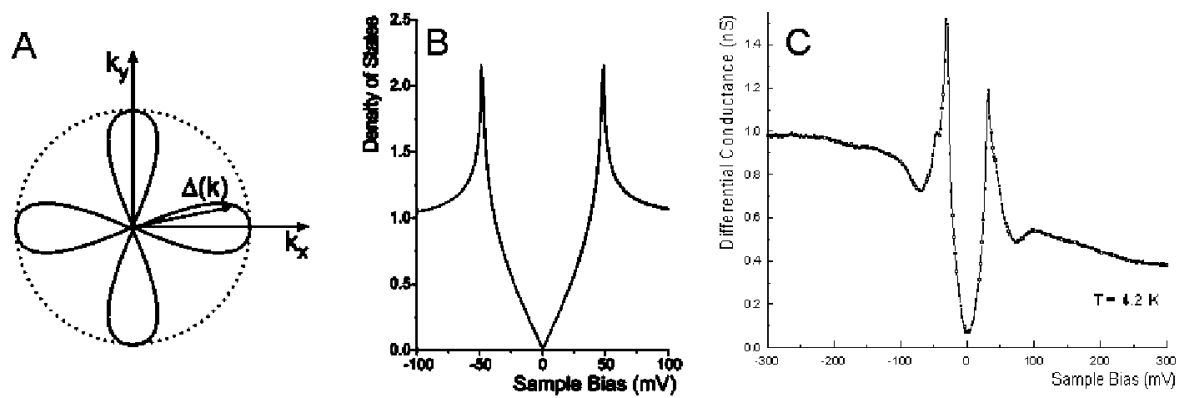

Fig. 4. (A) The momentum-space structure of a $d_{x^{2}-y^{2}}$ energy gap. (B) Differential conductance spectrum expected for a pure $d_{x^{2}-y^{2}}$ energy gap. (C) Measured differential conductance spectrum on the $\mathrm{BiO}$ surface of $\mathrm{Bi}-2212$.

LDOS-mapping is the process of measuring of $g(E)$ as a function of location $\boldsymbol{r}$ in a given field of view (FOV) to yield $g(\boldsymbol{r}, E)$ and thus energy-resolved local density of electronic states images $\operatorname{LDOS}(\boldsymbol{r}, E)$. If these $L D O S$ images have a large enough FOV and have high enough spatial resolution, they can be Fourier transformed to determine the $\boldsymbol{q}$-vectors associated with any spatial modulations 
of the LDOS. This is referred to as Fourier transform scanning tunneling spectroscopy [13] (FT-STS) and has recently been introduced to studies of the high- $T_{\mathrm{c}}$ cuprates [14]. In this technique, the wavelengths $\lambda$ (or wave vectors $q=2 \pi / \lambda$ ) of spatial modulations in $g(r, E)$ are identified by measuring the $\boldsymbol{q}$-vectors of local maxima in $g(\boldsymbol{q}, E)$, the Fourier transform magnitude of $g(r, E)$.

\section{Data}

We carried out measurements [15] of $g(r, E)$ in $\mathrm{a} \approx 650 \AA$ FOV spanned by $512^{2}$ pixels (or a spatial resolution of $1.3 \AA$ ). The sample-bias modulation is $2 \mathrm{mV}$ RMS. In Fig. 5a we show the topographic image, in $5 \mathrm{~b}$ an example of $g(\boldsymbol{r}, E)$, and in $5 c-$ h the magnitude Fourier transform $g(\boldsymbol{q}, E)$ of each $g(\boldsymbol{r}, E)$ at the labeled energy.

\section{Measuring the locus of scattering}

To motivate an understanding of these data, we considered a simple model based upon the electronic band structure. In Bi-2212 four nodes exist in the superconducting gap $\Delta(k)$ (Fig. $3 \mathrm{~B}$ ) and, below the gap maximum $\Delta_{0}$, the resulting contours in $k$-space along which quasiparticles exist at a given energy are "banana"-shaped (Fig. 3A). The quasiparticle density of states at energy $E=\omega, n(E=\omega)$, is proportional to

$$
\int_{E(k)=\omega}\left|\nabla_{k} E(\boldsymbol{k})\right|^{-1} \mathrm{~d} k
$$

where the integral is performed over the contour $E(k)=\omega$. Each "banana" evidently exhibits its largest rate of increase with energy, $\left|\nabla_{k} E(\boldsymbol{k})\right|^{-1}$, near its two ends. Therefore from Eq. (1), the primary contributions to $n(E)$ come from the octet of momentum-space regions centered around the points $\boldsymbol{k}_{j}(E) ; j=1,2, \ldots 8$, at the ends of the "bananas". An example of such an octet is shown as circles in Fig. $3 \mathrm{~A}$ and as dots in Fig. $6 \mathrm{~A}$.

In the presence of disorder, quasiparticles will be elastically scattered. A quasiparticle located in momentum-space near one element of the octet is then highly likely to be scattered to the vicinity of another element of the octet, because of the large density of final states there. Within this simple heuristic model, pairs of states at these momentum-space locations would then generate the most intense LDOS modulations.

At energy $E$, the wave vectors $\boldsymbol{q}_{i}(E)$ of these modulations would then be determined by all possible pairs of points in the octet $k_{j}(E)$. By considering one $\boldsymbol{k}_{j}$ in a representative octet in Fig. $4 \mathrm{~A}$ we see that there are seven characteristic scattering wave vectors $\boldsymbol{q}_{i}(E) ; i=1,2, \ldots 7$. This "octet"-model then predicts a total of $8 \times 7=56$ scattering wave vectors and, of these, 32 constitute a complete 


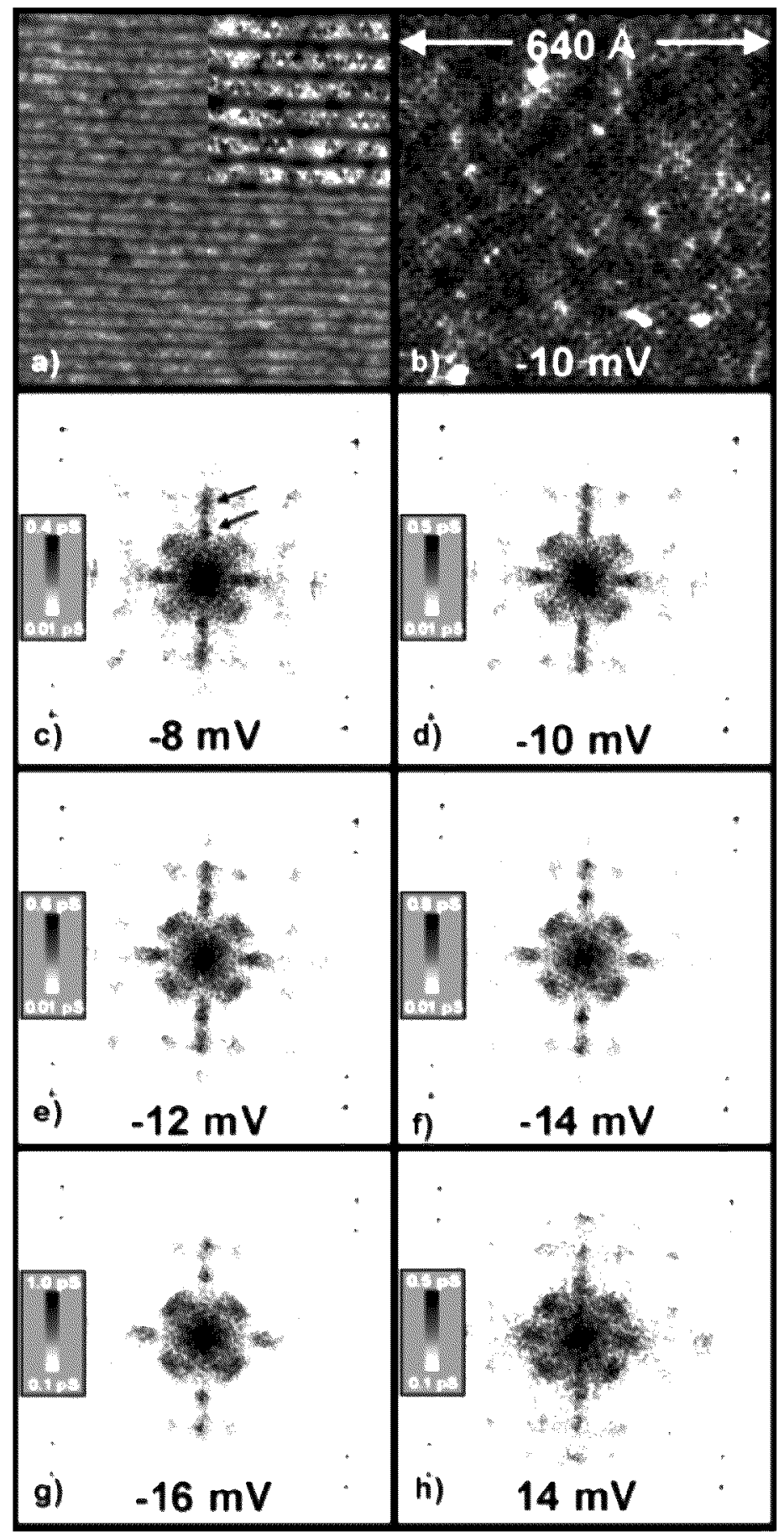

Fig. 5. (a) The topographic image of $\mathrm{BiO}$ surface in this FOV, (b) an example of $g(\boldsymbol{r}, E)$, and $(\mathrm{c}-\mathrm{h})$ the calculated $g(\boldsymbol{q}, E)$ for the labeled energies. 
set of inequivalent wave vectors - 16 pairs of which could be detected by FT-STS studies of Bi-2212.

Figure 5 shows that there are indeed numerous (up to 12 easily observable) inequivalent local maxima in $g(\boldsymbol{q}, E)$ as one would expect within the "octet"-model. By comparing the locations of these maxima at different energies, one can see that the $\boldsymbol{q}$-vectors do indeed disperse in different directions. In Fig. 6B we show the magnitudes of each of these interference wave vectors as measured from a complete set of data of the type in Fig. 5.
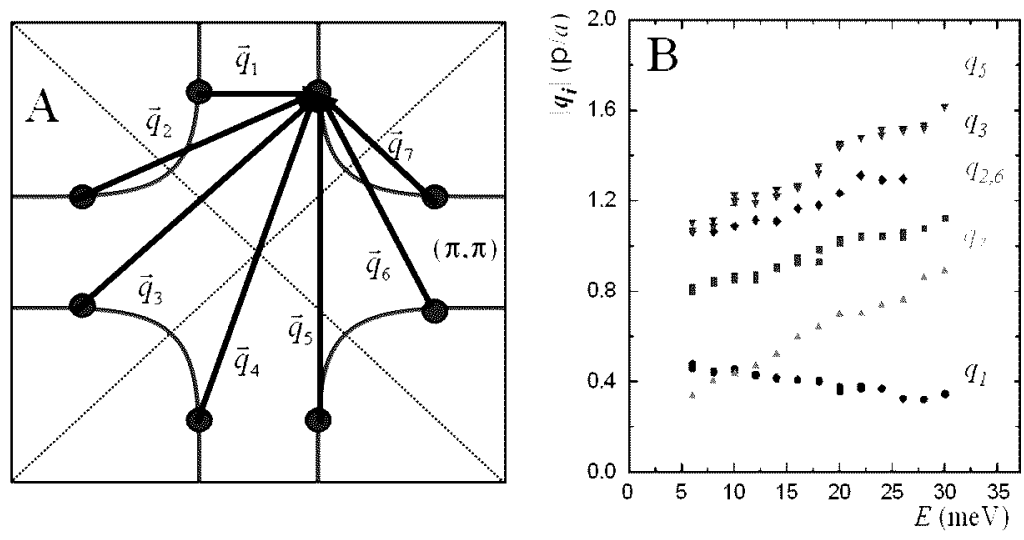

Fig. 6. (A) Representative set of seven scattering vectors $\boldsymbol{q}_{i}(E)$ of the "octet" model. (B) Measured dispersion for each of these $q$-vectors $\boldsymbol{q}_{1}$ to $\boldsymbol{q}_{7}$ (excluding $\boldsymbol{q}_{4}$ which we find difficult to observe).

Theoretical prediction of quasiparticle interference in high- $T_{\mathrm{c}}$ superconductors was made early in the literature [16]. More recent theoretical calculations [17-19] have confirmed the model that, at each energy, there will be up to 16 inequivalent sets of peaks in $\mid g(\boldsymbol{q}, \boldsymbol{\omega} \mid$, and each peak disperses with energy consistent with the quasiparticle dispersion $E(\boldsymbol{k})$. The data in Figs. 5 and 6 are in good qualitative agreement with these predictions. For example, between $-6 \mathrm{meV}>\omega>-30 \mathrm{meV}$, the locations of $\approx 50$ different $\boldsymbol{q}_{i}(\omega)$ can be identified as the wave vectors disperse.

From these data alone, the Fermi surface location $\boldsymbol{k}_{\mathrm{S}}$ and energy-gap $\Delta(\boldsymbol{k})$ can be estimated by assuming that each the measured $\boldsymbol{q}_{i}(\omega)$ are given by connecting vectors of the "octet" $k_{j}(\omega)$ at that energy. The result of $k_{\mathrm{S}}(\omega)$ for $6<|\omega|<30 \mathrm{meV}$ is shown as open circles in Fig. $7 \mathrm{~A}$. We interpret it as $k$-space trajectory of the ends of the energy-contour "bananas" or, in other words, the normal-state Fermi surface. In addition to the Fermi surface we also estimate the energy gap function $\Delta(\boldsymbol{k})$. For a given $\omega=e V$ we measure the associated $\boldsymbol{k}_{\mathrm{S}}$ thus yielding $\omega\left(k_{\mathrm{S}}\right)$. Within the heuristic octet model, this is the superconducting energy gap $\Delta\left(\boldsymbol{k}_{\mathrm{S}}\right)$ along the Fermi surface. We parameterize the location along $\boldsymbol{k}_{\mathrm{S}}$ by using the angle $\theta_{k}$ about the $(\pi, \pi)$ point. To compare the $k_{\mathrm{S}}(\omega)$ and $\Delta\left(\theta_{k}\right)$ from 

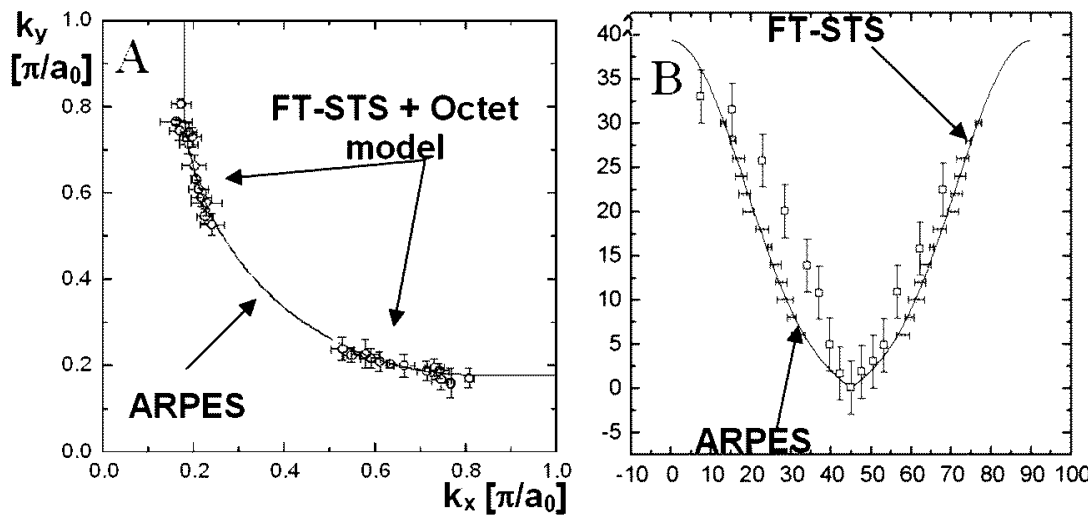

Fig. 7. (A) The locus of scattering measured from the data in Fig. 6B is shown as open circles. The ARPES-determined Fermi surface is estimated to be in the region of the gray band. (B) The FT-STS-derived momentum-dependent superconducting energy gap is shown as open circles while the ARPES-derived momentum-dependent energy gap is shown in open squares. They are in good agreement away from the antinodes where the interference patterns are not detectable.

FT-STS with those from ARPES, we plot the ARPES-determined Fermi surface as the gray band in Fig. 7A and the ARPES-derived $\Delta\left(\theta_{k}\right)$ in Fig. 7B as open squares.

The good agreement between these results derived from two totally different spectroscopic techniques, is a key result of this research project. The ARPES and STS results for Bi-2212 are demonstrably consistent with each other. Because the matrix elements for photoemission and tunneling are quite different, this agreement strengthens our confidence in both techniques. Furthermore, proposals that the widely reported [5, 6, 20-26] nanoscale electronic disorder in Bi-2212 might be due to surface damage not present in ARPES studies cannot be correct. This is because the momentum-space electronic structure as determined independently by both techniques is quite consistent. It therefore seems that STM and ARPES data are both correct and new theories are required for them to be reconciled with each other.

\section{Gapmap and antinodal quasiparticles}

Nanoscale disorder in the differential conductance spectra of Bi-2212 has been known for more than a decade [20, 21]. The first comprehensive atomically registered studies of the spatial arrangements in Bi-2212 tunneling spectra [22] were analyzed in terms of atomically-resolved maps of the superconducting energy gap in space or "gapmaps". These revealed several previously unknown characteristics including a $3 \mathrm{~nm}$ length scale characteristic patches within which the spectra remain quite uniform in gap magnitude but variable in amplitude. This 
phenomenology has by now been verified and explored by a wide range of new studies. These include studies of thin films [23], the relationship of topographic disorder to gapmap [5], effects of underdoping [6,24], effects of temperatures above $T_{c}[25]$, and effects of $\mathrm{Pb}$-doping [26]. The essential characteristics of this nanoscale electronic disorder of Bi-2212 is therefore highly repeatable and independent of sample preparation, cleave history, and STM/S instrumentation or technique. However, no microscopic explanation for these phenomena has so far been identified.

In Figs. 8 and 9 we show results from experiments relevant to this question. In Fig. 8A the gapmap measure in $\mathrm{a} \approx 14 \mathrm{~nm}$ FOV on an as-grown Bi-2212 surface is plotted using the color scale shown. It is quite consistent with those reported by other workers for as-grown samples. In Fig. $8 \mathrm{~B}$ is the LDOS map measured in this exact $\mathrm{FOV}$ at $E=24 \mathrm{meV}$. It is shown in a color-code so as to emphasize that wherever a $24 \mathrm{meV}$ gap magnitude is seen in Fig. 8A, a concomitant region of high

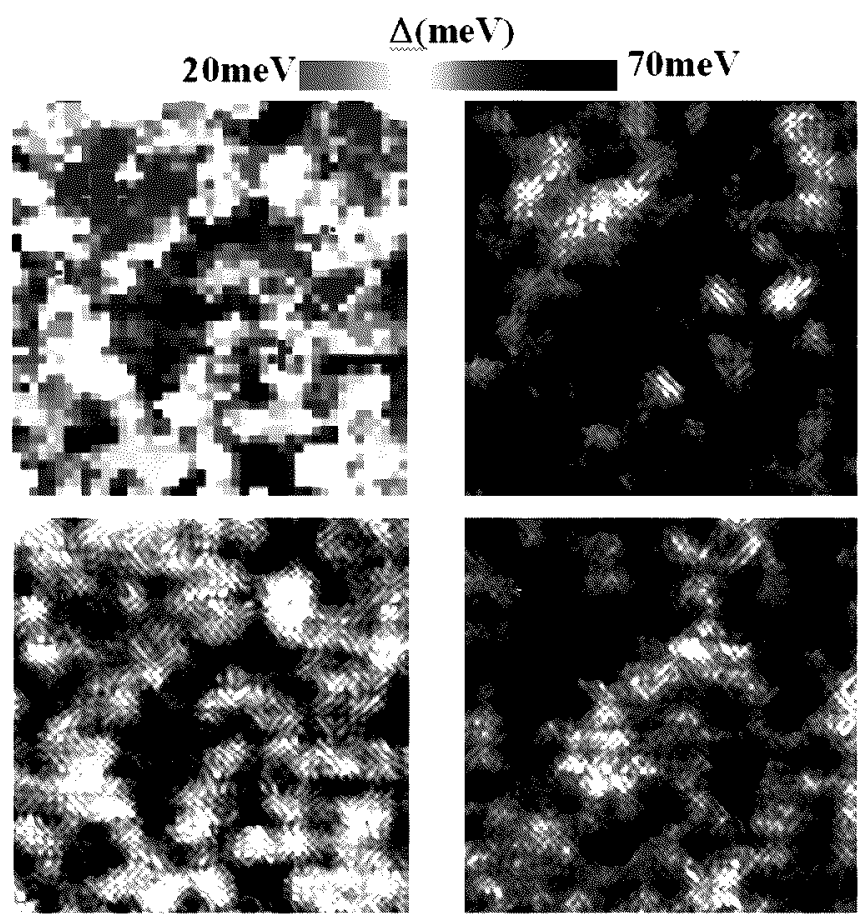

Fig. 8. (A) The gapmap measure in a $14 \mathrm{~nm}$ FOV on an as-grown Bi-2212 surface. (B) The LDOS map measured in this exact FOV at $E=24 \mathrm{meV}$. It is so color-coded to emphasize that wherever a $24 \mathrm{meV}$ gap magnitude is seen in (A), a concomitant region of high LDOS is seen in (B). (C) The LDOS map taken at $36 \mathrm{meV}$. Again, wherever a $36 \mathrm{meV}$ gap magnitude is seen in (A), a concomitant region of high LDOS is seen in (C). (D) The LDOS map taken at $54 \mathrm{meV}$. Wherever a $54 \mathrm{meV}$ gap magnitude is seen in (A), a concomitant region of high LDOS is seen in (D). 


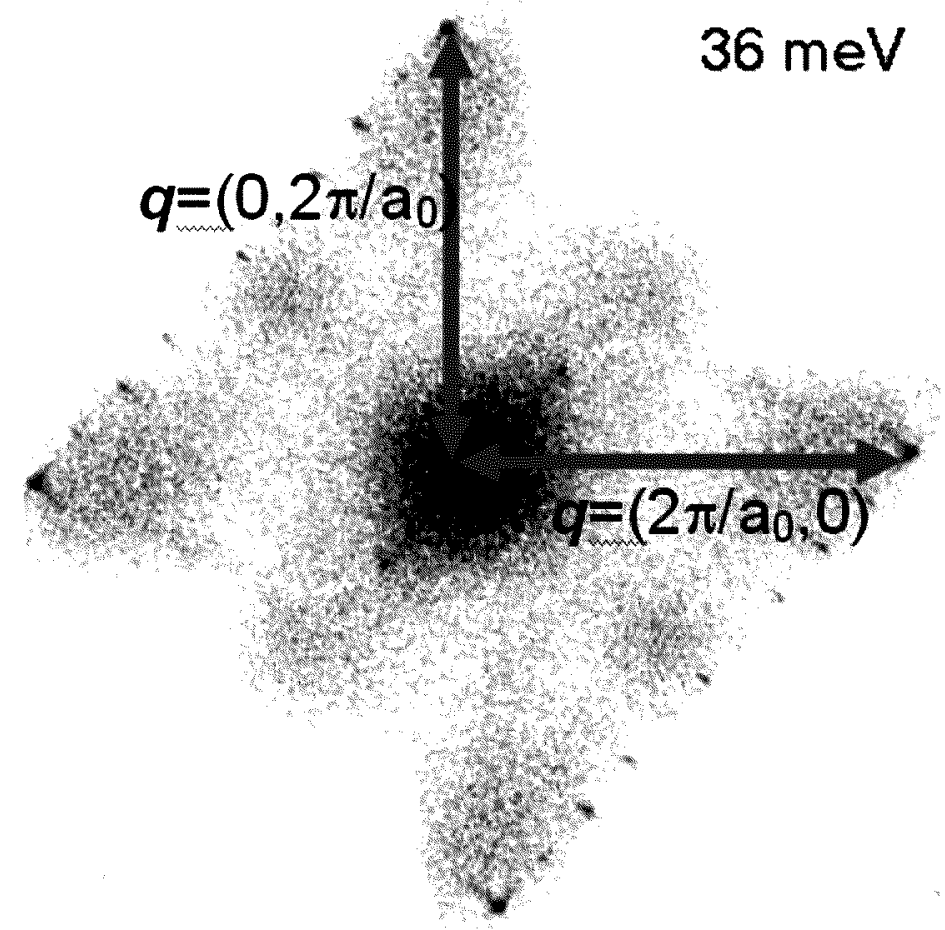

Fig. 9. (A) Fourier transform of LDOS map at $E=36 \mathrm{meV}$ which is $\approx \Delta_{0}$ for this sample. It is dominated by disorder at long wavelengths and intense peaks at $q=$ $(2 \pi / a, 0)$ and $q=(0,2 \pi / a)$.

LDOS with very short length scale modulations is seen in Fig. 8B. Similarly, the LDOS map taken at $36 \mathrm{meV}$ in Fig. $8 \mathrm{C} \mathrm{(54} \mathrm{meV:} \mathrm{Fig.} 8 \mathrm{D})$ has intense modulations of the same wave vector but now only where a $36 \mathrm{meV}$ ( $54 \mathrm{meV}$ ) gap magnitude is seen in Fig. 8A.

The transition from the long range $L D O S$-modulations in Fig. 5 to the very short range modulations in Fig. 8 occurs because, in real space, a rapid shortening of the range $L D O S$-modulations occurs as $\omega$ approaches $\Delta_{0}$, or equivalently as $\boldsymbol{k}_{j}$ approaches the Brillouin-zone-face. Even more surprisingly, all LDOS-modulations occurring at $\omega=\Delta_{0}$ (whatever the local value thereof) exhibit wave vector $\boldsymbol{q}=$ $\left(2 \pi / a_{0}, 0\right)$ or the equivalent. One can see an example of this in Fig. 9 which is the Fourier transform of the $36 \mathrm{meV}$ LDOS map and whose predominant features have wave vector $\boldsymbol{q}=\left(2 \pi / a_{0}, 0\right)$ or equivalent.

If we assume that these phenomena are occurring because the scattering is from $k=\left(\pi / a_{0}, 0\right)$ to $k=-\left(\pi / a_{0}, 0\right)$ and equivalent, one would deduce that, since $\Delta_{0}$ varies rapidly at the nanoscale, the energy condition for which $k=\left(\pi / a_{0}, 0\right)$ is satisfied at different locations for different energies. This further implies that 
local microscopic quasiparticle dispersions of antinodal quasiparticles differ on the nanoscale. This would be consistent with nanoscale carrier density variations [5].

\section{Future}

Key unresolved issues in FT-STS of Bi-2212 include: (1) whether the LDOS-checkerboard at the vortex core [27] is due to quasiparticle interference or to stabilization of another order parameter via suppression of superconductivity, and (2) how the relatively simple quasiparticle interference effects in near-optimal samples described herein, evolve with diminished doping as the material approaches the transition into the pseudogap region. New FT-STS experiments are required to address these issues.

\section{Acknowledgments}

The author wishes to acknowledge and thank all the collaborators on this project: H. Eisaki (AIST, Tsukuba), J.E. Hoffman (Stanford University), D.-H. Lee (U. of California, Berkeley), Kyle McElroy (U. of California, Berkeley), and S. Uchida (Tokyo University). We acknowledge and thank for research support: LASSP at Cornell University, Lawrence Berkeley National Lab., ONR Grant \# N00014-00-1-0066, NSF DMR\#9971502, NEDO, and by Grant-in-Aid for Scientific Research on Priority Area (Japan), a COE Grant from the Ministry of Education. J.E.H. acknowledges support of a Hertz Fellowship.

\section{References}

[1] A. Millis, J. Orenstein, Science 288, 5465 (2000).

[2] A. Damascelli, Z. Hussain, Z.X. Shen, Rev. Mod. Phys. 75, 473 (2003).

[3] P.D. Johnson, A.V. Fedorov, T. Valla, J. Electron Spectrosc. 117, 153 (2001).

[4] J.C. Campuzano, M.R. Norman, M. Randeria, preprint available at cond-mat/0209476.

[5] S.H. Pan, J.P. O’Neal, R.L. Badzey, C. Chamon, H. Ding, J.R. Engelbrecht, Z. Wang, H. Eisaki, S. Uchida, A.K. Gupta, K.-W. Ng, E.W. Hudson, K.M. Lang, J.C. Davis, Nature 413, 282 (2001).

[6] K.M. Lang, V. Madhavan, J.E. Hoffman, E.W. Hudson, H. Eisaki, S. Uchida, J.C. Davis, Nature 415, 412 (2002).

[7] D.S. Dessau, Z.X. Shen, D.M. King, D.S. Marshall, L.W. Lombardo, P.H. Dickinson, A.G. Loeser, J. DiCarlo, C. Park, A. Kapitulnik, W.E. Spicer, Phys. Rev. Lett. 71, 2781 (1993).

[8] P. Aebi, J. Osterwalder, P. Schwaller, L. Schlapbach, M. Shimoda, T. Mochiku, K. Kadowaki, Phys. Rev. Lett. 72, 2757 (1994).

[9] B.O. Wells, Z.X. Shen, D.S. Dessau, W.E. Spicer, D.B. Mitzi, L. Lombardo, A. Kapitulnik, A.J. Arko, Phys. Rev. B 46, 11830 (1992). 
[10] Z.-X. Shen, D.S. Dessau, B.O. Wells, D.M. King, W.E. Spicer, A.J. Arko, D. Marshall, L.W. Lombardo, A. Kapitulnik, P. Dickinson, S. Doniach, J. DiCarlo, T. Loeser, C.H. Park, Phys. Rev. Lett. 70, 1553 (1993).

[11] H. Ding, M.R. Norman, J.C. Campuzano, M. Randeria, A.F. Bellman, T. Yokoya, T. Takahashi, T. Mochiku, K. Kadowaki, Phys. Rev. B 54, R9678 (1996).

[12] Z.-X. Shen, W.E. Spicer, D.M. King, D.S. Dessau, B.O. Wells, Science 267, 343 (1995).

[13] L. Petersen, Ph. Hofmann, E.W. Plummer, F. Besenbacher, J. Electron Spectrosc. Relat. Phenom. 109, 97 (2000); D. Fujita, K. Amemiya, T. Yakabe, H. Nejoh, T. Sato, M. Iwatsuki, Surf. Sci. 423, 160 (1999).

[14] J.E. Hoffman, E.W. Hudson, K.M. Lang, V. Madhavan, H. Eisaki, S. Uchida, J.C. Davis, Science 295, 466 (2002).

[15] K. McElroy, R.W. Simmonds, J.E. Hoffman, D.-H. Lee, J. Orenstein, H. Eisaki, S. Uchida, J.C. Davis, Nature 422, 592 (2003).

[16] J.M. Byers, M.E. Flatté, D.J. Scalapino, Phys. Rev. Lett. 71, 3363 (1993).

[17] Q.H. Wang, D.H. Lee, Phys. Rev. B 67, 020511 (2003).

[18] A. Polkovnikov, S. Sachdev, M. Vojta, Physica C 388, 19 (2003).

[19] D.G. Zhang, C.S. Ting, Phys. Rev. B 67, 100506 (2003).

[20] J.-X. Liu, J.-C. Wan, A.M. Goldman, Y.C. Chang, P.Z. Jiang, Phys. Rev. Lett. 67, 2195 (1991).

[21] A. Chang, Z.Y. Rong, Y.M. Ivanchenko, F. Lu, E.L. Wolf, Phys. Rev. B 46, 5692 (1992)

[22] V. Madhavan, K.M. Lang, E. Hudson, S.H. Pan, H. Eisaki, S. Uchida, J.C. Davis, Bull. Am. Phys. Soc. 45, 416 (2000).

[23] C. Howald, P. Fournier, A. Kapitulnik, Phys. Rev. B 64, 100504 (2001).

[24] T. Cren, D. Roditchev, W. Sacks, J. Klein, Europhys. Lett. 54, 84 (2001).

[25] A. Matsuda, T. Fujii, T. Watanabe, Physica C 388, 207 (2003).

[26] G. Kinoda, T. Hasegawa, S. Nakao, Phys. Rev. B 67, 224509 (2003).

[27] J.E. Hoffman, K. McElroy, D.-H. Lee, K.M. Lang, H. Eisaki, S. Uchida, J.C. Davis, Science 297, 1148 (2002). 